\title{
HPV Vaccine for Prevention of Cervical Cancer: Key Issues and Challenges for Developing Countries
}

Human papillomavirus (HPV) is now recognized as the major cause of cervical cancer, which is the second most common cancer in women worldwide, and the principal cancer of women in most developing countries, where 80 percent of cases occur. ${ }^{1}$

It is also a major cause of morbidity and mortality in Nepal. As in other countries in South Asia and developing countries worldwide, it causes $34 \%$ of all cancers in women. ${ }^{2}$ A Pap test (cervical cytology screening) is the best way to detect cell changes, which may be an early sign of pre-cancer of the cervix.

HPVs are a group of more than 100 related viruses that usually infect soft tissue and epidermis of both male and female genitalia. Genital HPV infections are very common in human, and are sexually transmitted. Most HPV infections are asymptomatic and resolve spontaneously. However, HPV infection sometimes persists for many years. Such infections are the primary cause of cervical cancer. ${ }^{3}$ HPVs may also play a role in cancer of the anus, vulva, vagina, penis, as well as oro-pharynx. More than 30 types of HPV can infect the genital area of people. About 15 sexually transmitted HPVs are referred to as being "high-risk" because they are more likely to lead to cancer, and these include types $16,18,31,33,35,39,45,51,52,56,58,59,66,68$, and 73. A few types of HPV are also known to cause warts.

A new vaccine, a great breakthrough of the $21^{\text {st }}$ century, is available that protects against four types of HPV causing the most cases of cervical cancer and genital warts. Gardasil ${ }^{\circledR}$, a quadrivalent HPV vaccine that is highly effective in preventing infection with four types of HPV, is approved by FDA/US for females aged between 9-26 years. ${ }^{4}$ Two of these four HPVs (16 and 18) cause about 70 percent of cervical cancers ${ }^{5}$, and the other two HPVs (6 and 11) cause about 90 percent of genital warts. This vaccine is most effective if it is given before a woman is infected with HPV. Therefore, it is best for a woman to get the vaccine before she is sexually active. The vaccine is not recommended for pregnant women, however, it is safe for lactating women. It is less likely to have serious sideeffects. $^{4}$

Presently, the vaccine costs approximately US $\$ 360$ for a set of three doses. Here, the second and third doses should be given two and six months after the first dose respectively. The vaccine does not protect against all types of HPV. Therefore, women who are vaccinated should still undergo regular Pap tests. The vaccine is a preventive tool and is not a substitute for cancer screening.

Even though the vaccine protects against HPV associated cervical cancer, many adolescents are reluctant and their parents not endorsing the vaccine due to low acceptability and high cost. Similarly the society also perceives cervical cancer as a problem of women, believing there to be minimal or no role of men in transmitting the virus to women. To prevent HPV infection among females, males should be vaccinated as well.

One of the greatest barriers to the introduction of this vaccine is its cost. Dramatic cost reduction will be required to facilitate its timely use in developing countries. With subsidies from donors, HPV vaccine can be made available to the poor women of the world.

Since the vaccines have become available, mandated vaccination has become a highly debatable and controversial issue. The approval of the vaccine against cancer-causing HPV strains is significant in public health, but more so in developing countries lacking 
health care resources for routine cervical cancer screening. There are disputes over who to vaccinate and whether it should become a national program run by the government or paid by the individuals. The mass administration of a HPV vaccine provides a great potential for improving the health of millions of women as well as men.

Efforts must also be made to educate the public about the pervasiveness of HPV and the advantages of vaccination. One strategy would be to perform universal vaccination so that no one feels stigmatized. However, achieving broad coverage of adolescents and securing financing will be a challenge in the developing countries.

These benefits of vaccination are obvious for the developed world. However, women in developing countries may benefit even more. Considerations for policymakers debating the use of HPV vaccine in any particular country will include country's disease burden, its health care infrastructure, and its capacity for initiating and sustaining an immunization program for adolescents. Other considerations include the affordability and cost-effectiveness of vaccination relative to other programs competing for resources and the likelihood of cultural acceptability, political will, and public support. ${ }^{6}$

The single-most barrier to the introduction of the vaccine for the adolescents is its price. From public health perspective, the first priority in resource-poor settings would be to vaccinate young adolescent girls through school-based or community based vaccination programs.

Therefore, everyone should be highly committed to make extensive efforts in utilizing this new armamentarium of the modern medicine so as to prevent cervical cancer.

\section{REFERENCES}

1. Ferlay J, Bray F, Pisani P, Parkin DM. Globocan 2002: cancer incidence, mortality and prevalence worldwide. IARC CancerBase no. 5, version 2.0. Lyon, France: IARC Press; 2004

2. The International Network for Cancer Treatment and Research. Perspectives in cervical cancer prevention in India. [Online]. [Cited 2009 Sep 05]. Available from: URL: http://www.inctr.org/ publications/2003_v03_n03_w02.shtml

3. Schiffman M, Castle PE. Human papillomavirus: epidemiology and public health. Archives of Pathology and Laboratory Medicine. 2003;127(8):930-4.

4. National Cancer Institute. HPV (human papillomavirus) vaccines for cervical cancer. [Online]. [Cited 2009 Sep 05]. Available from: URL: http://www.cancer.gov/cancertopics/hpv-vaccines

5. Munoz N, Bosch FX, Castellsague $X$ et al. Against which human papillomavirus types shall we vaccinate and screen? The international perspective. International Journal of Cancer. 2004;111:278-85.

6. Agosti JM, Goldie SJ. Introducing HPV Vaccine in developing countries - key challenges and issues. N Engl J Med. 2007;356(19):1908-10. 